\title{
3D rotational DSA: Diagnostic and therapeutic approach to cerebral aneurysms
}

\author{
Karuna Tamrakar Karki, ${ }^{1}$ Duan Chuan Zhi ${ }^{1}$, Li Tie Lin ${ }^{1}$ \\ ${ }^{1}$ Southern Medical University, Department of Neurosurgery, Zhujiang hospital. \\ Correspondence: \\ Dr Karuna Tamrakar Karki \\ Department of Neurosurgery, B \& C Medical College and Teaching Hospital \& \\ Research Center,Birtamode,Jhapa,Nepal. \\ Email: tamrakarkaruna@gmail.com \\ Phone: +9779843405124
}

Background and purpose: Digital subtraction angiography (DSA), a minimally invasive procedure for an exceptional visualization of cerebrovascular angioarchitecture, has become an important imaging technique for inclusive evaluation and therapeutic embolization. Our purpose is to share the importance of DSA for evaluation of cerebral aneurysms. Material and method: A total of 148 patients with suspected intracranial aneurysm underwent digital subtraction angiography during January 2005 to December 2009. Preliminarily aortogram was considered in old age group (>50yrs) before supraaortic angiography. Patient's selection criterion included both ruptured and unruptured aneurysms. 3D rotational effect was applied for all complex cerebral aneurysms with unfavorable dome neck ratio. DSA was accomplished into 2- 6 standard projections for every vascular territory for optimal visualization and accurate evaluation of cerebral aneurysms. Result: 21 were males and 48 were females. Age difference was ranged between 26-78 years with an average of 51.5years. Among 288 patients, total detected aneurysms were 480. 32 presented with SAH, 21 presented with headache and 14 patients came with cranial nerve dysfunction during admission. 3 had negative angiography irrespective to spontaneous subarachnoid hemorrhage. Delineation of aneurysmal neck improved with rotation effect of 3D DSA in $80 \%$ of patients. Parent vessel and its relationship to adjacent vessels was better demonstrated with rotational angiography in $>50 \%$ of cases. Endovascular embolization was done in 80 patients in same setting. Conclusion: DSA with 3D rotational technique is an essential radio-imaging tool for accurate characterization of cerebrovascular diseases and subsequently beneficial to elaborate on their potential treatment protocols.

Key words: Digital subtraction angiography; 3D rotational technique; cerebral aneurysms

$\mathrm{D}$ igital subtraction angiography (DSA) technique was $1^{\text {st }}$ described by Zides des Plantes in early $1935^{1}$. Zilkha et al in $19761^{\text {st }}$ used the system of computerized subtraction for the assessment of regional cerebral blood flow in which pre-injection scans were subtracted from post-injection 3

Date submitted: 7/ 8/2019

Date accepted: 9/9/2019 scans for analysis of scanned section ${ }^{2}$. Kruger et al in 1979 described computerized fluorography in which images were logarithmically collected, amplified and digitalized before being integrated, processed and redisplayed on a monitor ${ }^{3}$. Consequently from mid 90s, exploitation of 3D digital

egneuro Volume 01, Issue 02, 2019

DOI: https://doi.org/10.3126/egn.v1i2.25726 
reconstruction and rotational imaging technique has gradually become popular for diagnosis of vascular lesions in clinical practice.

\section{Material and Methods}

A total of 288 patients with suspected intracranial aneurysm underwent digital subtraction angiography during January 2005 to December 2010. Before supra-aortic angiography, preliminarily aortogram was considered in every elderly patient (> 50 years). Patient's selection criterion included both ruptured and unruptured aneurysms. 3D rotational effect was applied to all complex cerebral aneurysms with unfavorable dome neck ratio. DSA was accomplished into 2- 6 standard projections for every vascular territory for optimal visualization and accurate evaluation of cerebral aneurysms. .

\section{Exclusion criteria}

The study excluded patients treated with surgical clipping of cerebral aneurysms.

\section{D and 3D image reconstruction technique}

DSA examination was performed under AXIOM Artis, C arm unit (Siemens Medical System, Germany). Inform consent was obtained from all patients. Under sedation and local anesthesia, 4-vessel angiography (bilateral internal carotid and vertebral) was performed via femoral access. 5F angiographic catheter was selectively navigated into desired vessel. This study included at least three projections (anterior-posterior, lateral and oblique) and rotational technique for 3D reconstruction images. For each projection, 5$7 \mathrm{ml}$ of nonionic contrast bolus dose of Ioversol (320 $\mathrm{mg} / \mathrm{ml}$ ) was injected via a pressure injector (Medrad, USA) at the rate of $3-4 \mathrm{ml} /$ sec to obtain 3-4 frames per second to late venous phase. For 3D rotation angiography mode, over an $180^{\circ}-200^{\circ}$ angle was used with a maximum rotation speed between $20^{\circ}-30^{\circ}$ per second. $15 \mathrm{ml}$ of contrast medium was injected at the rate of $3 \mathrm{ml} / \mathrm{sec}$. 3D images were reconstructed from data collected from rotational arc in a workstation using Syngo software program. Magnification views were assimilated to elucidate significant findings.

\section{Results}

Among 288 patients, total aneurysm documentation number was 480. 3 had negative angiography irrespective to spontaneous subarachnoid hemorrhage. Delineation of aneurysmal neck improved with rotation effect of 3D DSA in $80 \%$ of patients. Parent vessel and its relationship to adjacent vessels was better demonstrated with rotational angiography in $>50 \%$ of cases. Endovascular embolization was done in 80 patients in same setting.

In total 45 aneurysms were located in ICA, 15 were anterior communicating aneurysms 11 were at middle cerebral artery, 49 were detected in anterior communicating artery and 12 were demonstrated in vertebrobasilar circulation.

78 had single aneurysm 34 had double aneurysm 4 had three aneurysms. $89 \%$ of multiple aneurysms were detected in anterior circulation.

Size of the aneurysmal sac detected by DSA ranged from $3.5 \mathrm{~mm}$ to $30.0 \mathrm{~mm}$ with mean size of $6.2 \mathrm{~mm}$. 62 of the aneurysms were $<5$ $\mathrm{mm}$, other 62 aneurysms ranged between $6 \mathrm{~mm}$ and $15 \mathrm{~mm}, 7$ aneurysms were $16 \mathrm{~mm}$ to 25 $\mathrm{mm}$ and 1 was $>25 \mathrm{~mm}$.

Size of aneurysmal neck ranged from $1.8 \mathrm{~mm}$ to $12.2 \mathrm{~mm}$ with mean of $4.3 \mathrm{~mm}$. Aneurysmal size measured in 3D rotational angiography found to be a bit larger than measured by conventional DSA. Although 3D effect was only applied in complex shaped cerebral aneurysms, comparison of every aneurysmal 
configuration could not be possible by conventional DSA and rotational 3D DSA.

In 132 aneurysms, DSA demonstrated 8 aneurysms with branching arteries arising from the aneurysmal sac. 3 were MCA aneurysms, 2 Acoma, 2 PComA and 1 PCA aneurysm. In 5 aneurysm vessel branching was demonstrated in neck region and other branching arteries were revealed from aneurysmal dome.

Endovascular treatment comprised coiling alone or stent-assisted coiling for effective obliteration and for better outcome. 24 patients were treated early ( $>24$ hours). 43 patients had endovascular treatment in 24 to 72 hours and 63 aneurysms were embolized after 72 hours of discovery in DSA.

Initial diagnostic angiography procedure related complication was nil.

\section{Discussion}

DSA with 3D reconstruction and rotational imaging technique provides detail information regarding anatomical configuration of intracranial aneurysms, accurate measurements, relationship of the parent vessels and collateral flow competence. These characteristic features are essential for further management either by endovascular therapy or by surgical clipping.

Giant and fusiform aneurysms: Aneurysms which are found at difficult locations and cranial base including giant and fusiform lesions are termed as complex intracranial aneurysms. These are associated with high risk of subarachnoid hemorrhage (SAH), and progressive neurological deterioration or death, caused by mass effect on the surrounding brain tissue or stroke, caused by emboli that dislodges to main vascular territory ${ }^{4}$.Giant intracranial aneurysms (fig1 A) constitute only $5 \%$ of all intracranial aneurysms which significantly occur in pediatric age and in elderly women ${ }^{4}$. Internal carotid artery egneuro Volume 01, 2019 (cavernous and paraclinoid) is frequently involved followed by vertebrobasilar circulation. Thus detail
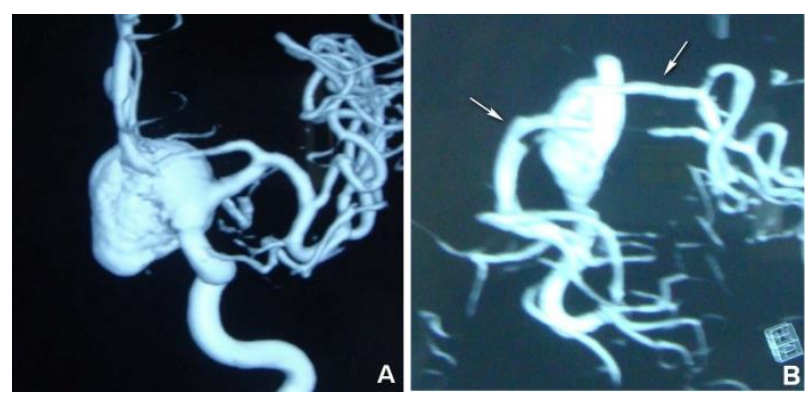

Figure 1: a 65-year-old lady with progressive loss of vision. A: ICA arteriography (3D rotational image) demonstrates giant supraclinoid ICA aneurysm. B: 3D rotational DSA of vertebrobasilar circulation shows giant fusiform aneurysm of BA and both PCAs originates from the aneurysm sac (white arrows).

preoperative evaluation including selective catheter based cerebral angiography with 3D rotation reconstruction images are necessary before any surgical intervention. Giant and fusiform aneurysms have higher rate of arterial wall calcification, atherosclerotic plaque and intra-luminal thrombus which usually have tendency to complicate the surgical management. Fusiform aneurysms (fig1B) are generally found in vertebrobasilar system mostly affecting basilar artery in elderly age group. Laminated thrombus is usually a positive sign suggesting repeated episodes of partial thrombosis 5. Atherosclerosis and hypertension are main cause for the fusiform dilation of intracranial or extracranial arteries. ${ }^{5}$

Multiple aneurysms: Multiple intracranial aneurysms (fig 2) are usually seen in one third of the patients with cerebral aneurysms ${ }^{6}$. In a series of 254 patients, multiple aneurysms were detected angiographically almost in one half of the cases $(44.9 \%)^{7}$. Anterior circulation is commonly involved territory to develop multiple intracranial aneurysms. Ipsilateral occurrence of multiple lesions is greater than 
in contralateral side. Vertebrobasilar system is less commonly involved territory for multiple aneurysmal growths. Four vessels cerebral angiography has become mandatory for acute search of multiple lesions when a patient harbors aneurysm in ICA or in pericallosal artery.
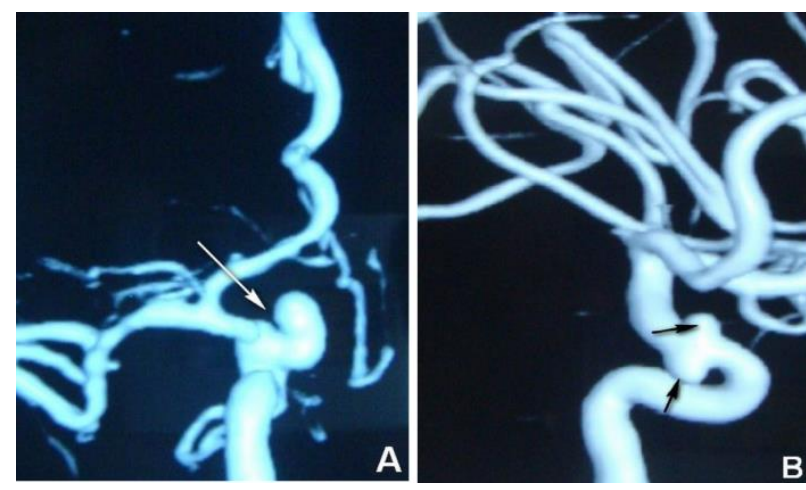

Figure 2: a 44-year-old lady underwent DSA for suspected aneurysmal SAH. A: Selective right ICA arteriography in lateral projection detects $6 \mathrm{~mm} \mathrm{x}$ $4 \mathrm{~mm}$ aneurysm arising from supraclinoid ICA. B: left ICA 3D rotational angiogram demonstrates additional two small aneurysms at cavernous ICA.

Dissecting aneurysms: DSA has remains the gold standard for evaluation of dissecting aneurysms (fig 3). A wide spectrum of angiographic findings has been reported, such as a pearl and string sign 8,9 , a tapered narrowing or string sign ${ }^{8}$, a total occlusion with proximal distention ${ }^{9}$ or out-pouching variety ${ }^{10}$. Among these findings, the most commonly detected type is pearl and string sign, which has often been mistaken for a saccular aneurysm associated with vasospasm 9,10 . Therefore, an angiographic identification of double lumen, representing the passage of contrast medium into both true and false channels, is essential for a decisive diagnosis of dissecting aneurysms. Known the less, coiling of such aneurysm is more challenging (Fig 3B)
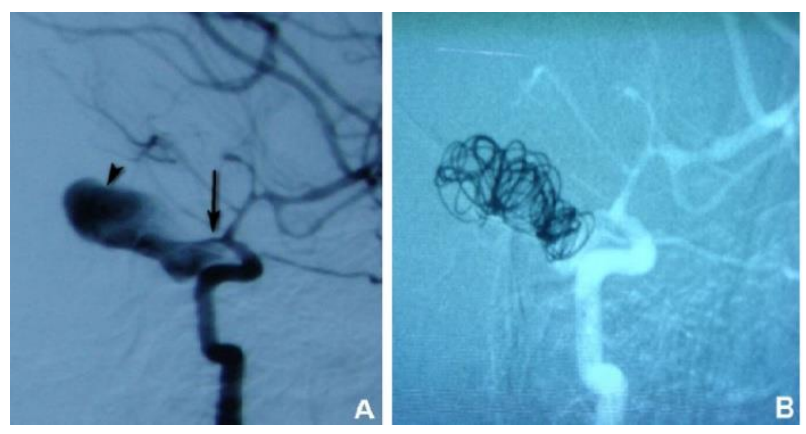

Figure 3: Subtracted image of a 38-year-old man with aneurysmal SAH. A: In selective right ICA angiography, out pouching variety (arrow head) of large dissecting aneurysm is detected originating from supraclinoid ICA in oblique view. B: Coiling being performed on roadmap view of same patient.
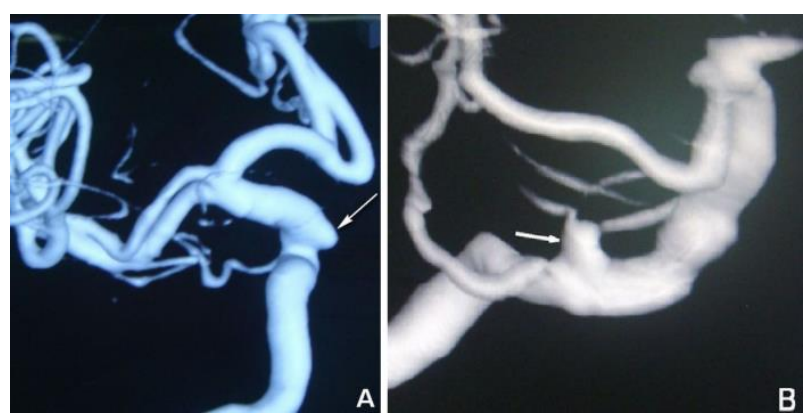

Figure 4: a 45-year-old lady with chronic headache. A: In ICA arteriogram, showing $1 \mathrm{~mm} x$ $2 \mathrm{~mm}$ sized aneurysm (arrow) arising from supraclinoid left ICA (3D reconstruction and rotational image). B: Hypertensive elderly man with $\mathrm{SAH}$, vertebral angiogram demonstrates $2 \mathrm{~mm}$ $x$ 3mm sized wide neck aneurysm (arrow) at the close proximity of vertebrobasilar junction (3D reconstruction and rotational image).

Micro-aneurysms: The terms "microaneurysm" and "very small aneurysm" have both been used to describe aneurysms smaller than $3 \mathrm{~mm}$ in size. In DSA imaging system, hidden or overlapped bony structures are subtracted for better image enhancement allowing detection of micro-aneurysms of less than $3 \mathrm{~mm}$ in size (fig 4) which is yet not possible with MR angiography. Moreover, incidence of micro-aneurysms is higher at 
anterior communicating artery as compared with other locations of circle of Willis ${ }^{11}$.

\section{Conclusion}

Recent technical advances made in DSA including 3D rotational technique are the essential radio-imaging tools giving better spatial resolution images immediately, and chances of vessels superimposition and degradation of image quality is less as compared to conventional angiography. Being an essential part of neurointerventional technology, it remains the study of choice for precise interpretation of angioarchitecture of various neurovascular diseases and subsequently to elaborate on the potential treatment protocols.

\section{References}

1. Zides des Plantes. BG: subtraktion. Eine rontgenographische methode zur separaten abbildung bestimmer teile des objekts. Fortschr Roentgenstr. 1935;52:69-79

2. Zilkha E, Ladurner G, Miff LD, Du Boulay GH and Marshall J. Computer subtraction in regional cerebral blood-volume measurements using the EMI-scanner. BJR. 1976; 49: 330-4.

3. Kruger RA, Mistretta CA, Houk TL, Riederer SJ, Shaw CG. Computerized fluoroscopy in real time for noninvasive visualization of the cardiovascular system-preliminary studies. Radiology. 1979;130:49-57.

4. Barrow DL, Alleyne C. Natural history of giant intracranial aneurysms and indications for intervention. Clin Neurosurg. 1995;42:214-44.

5. Shokunbi MT, Vinters HV, Kaufmann JCE. Fusiform intracranial aneurysms: Clinicopathologic features. Surg Neurol. 1988;29:263-70.

6. Rinne J, Hernesniemi J, Puranen M, Saari T. Multiple intracranial aneurysms in a defined population: prospective angiographic and clinical study. Neurosurg. 1994;35:803-8.
7. Wilson FMA, Jaspan T, Holland IM. Multiple cerebra aneurysms-A reappraisal. Neuroradiology. 1989;31:232-6.

8. Ojemann RG, Fisher CM, Rich JC. Spontaneous dissecting aneurysm of internal carotid artery. Stroke. 1972;3:434-40.

9. Yonas H, Agamanolis D, Takaoka Y, White RJ. Dissecting intracranial aneurysms. Surg Neurol. 1977;8:407-15.

10. Waga S, Fujimoto K, Morooka Y. Dissecting aneurysm of the vertebral artery. Surg Neurol. 1978;10:237-9.

11. Birknes JK, Hwang SK, Pandey AS, Cockroft K, Dyer AM, Benitez RP, Veznedaroglu E, Rosenwasser RH: Feasibility and limitations of endovascular coil embolization of anterior communicating artery aneurysms: Morphological considerations. Neurosurgery 2006;59:43-52. 